\title{
Incisional hernia repair: still a complex matter
}

\author{
G. Campanelli ${ }^{1}$
}

Published online: 31 March 2021

(c) The Author(s), under exclusive licence to Springer-Verlag France SAS, part of Springer Nature 2021

Laparoscopy and, more recently, the use of robotic techniques in general surgery have improved the post-op course of numerous diseases. This improvement can be attributed to greater precision, due to enhanced vision of the surgical field, optimal "management" of small spaces, the possibility of safely identifying even the smallest structures, and not least the minimal destruction of the abdominal wall (with incisions sometimes measuring only a few centimeters).

Nevertheless, the incidence of incisional hernia remains high.

There are two reasons for this: the still high rate of open surgery, and the fact that defects (including hernias) can also develop at lap/robotic access points.

Notably, many of these patients, despite the small incision used for the primary procedure, go on to develop complex incisional defects that increase in size rapidly and dramatically, creating sometimes complex situations.

Moreover, rising rates of obesity, together with often excessively long waits for abdominal wall surgery, have exacerbated this situation. We are seeing more and more complex cases, where both the surgical option itself, not to mention the management of possible post-surgical complications, present major challenges.

This situation has led the idea to take root, among patients and surgeons, that a super specialization needs to be created in order to ensure that cases of this kind are approached adequately.

Furthermore, the time now seems to be ripe for hospitals/ clinics/universities, too, to address the need for ad hoc centers and formal and institutional specializations for surgeons wishing to devote themselves to this surgical field.

With the aim of attracting surgeons to the field of hernia, surgical scientific societies the world over have long being organizing, at national or continental level, expertrun schools and training opportunities aimed at general surgeons, such as "web masters" courses that, through the sharing of experience, aim to facilitate decision making and clarify surgical approaches.

As confirmation of these developments, the European Commission has now started issuing the first "certificates" to the FEBS (Fellows of the European Board of SurgeryAbdominal Wall Surgery) of the European Union of Medical Specialists (UEMS).

Our journal, which is a true beacon in the field of science-driven surgical approaches thanks to the caliber of our authors, strongly urges a movement in this direction.

Publisher's Note Springer Nature remains neutral with regard to jurisdictional claims in published maps and institutional affiliations.

G. Campanelli

hernia.editorialoffice@gmail.com

1 University of Insubria, Gruppo Ospedaliero San Donato, Milan, Italy 